\title{
Angiolymphoid Hyperplasia with Eosinophilia with Clinical Presentation Concerning for Juvenile Temporal Arteritis
}

\author{
Emily Li ${ }^{a} \quad J o h n$ Sinard ${ }^{a}$ b Alberto Distefano ${ }^{c}$ Mahsa Sohrab ${ }^{a}$ \\ a Yale Eye Center, Department of Ophthalmology and Visual Science, Yale School of Medicine, New Haven, CT, USA; \\ ${ }^{b}$ Department of Pathology, Yale School of Medicine, New Haven, CT, USA; ' Boston Medical Center, Department of \\ Ophthalmology, Boston University School of Medicine, Boston, MA, USA
}

\section{Established Facts}

- Angiolymphoid hyperplasia with eosinophilia (ALHE) is a rare, benign vascular tumor that characteristically presents in the head and neck of females (70\%) in their 3rd to 5th decade of life.

- ALHE lesions may be asymptomatic or may be associated with pruritis, pain, and/or bleeding. They are typically firm and nontender on examination.

- ALHE typically appears on magnetic resonance imaging as a well-defined, isointense mass that enhances with gadolinium contrast, with or without an associated vascular pedicle.

\section{Novel Insights}

- ALHE can present in the adolescent population and should be included in the differential diagnosis of pediatric vascular tumors of the head and neck.

- The clinical and radiographic presentation of ALHE can resemble juvenile temporal arteritis. Definitive diagnosis requires histopathological evaluation.

\section{Keywords}

Adnexal tumors · Eyelid · Histopathological study Oculoplastic surgery · Pediatrics · Tumors

\section{Abstract}

A 20-year-old boy presented with a mass superotemporal to the right eyebrow of 1 month's duration without a history of recent trauma. Complete examination and workup, including laboratory analysis, Doppler ultrasound imaging, and magnetic resonance imaging, suggested a diagnosis of juvenile temporal arteritis. Excisional biopsy and histopathology contradicted the preoperative workup and yielded the final diagnosis of angiolymphoid hyperplasia with eosinophilia, a rare eosinophilic dermatosis of unknown etiology. This case highlights the need for better characterization of temporal vascular tumors in the pediatric population.

(c) 2019 S. Karger AG, Basel

\section{Introduction}

Angiolymphoid hyperplasia with eosinophilia (ALHE), also known as epithelioid hemangioma (EH), is a rare, benign vascular tumor of unknown etiology. Lesions represent localized proliferations of capillaries

\section{KARGER}

(c) 2019 S. Karger AG, Basel

E-Mail karger@karger.com

www.karger.com/oop
Emily Li, MD

Yale Eye Center, Department of Ophthalmology and Visual Science

Yale School of Medicine, 40 Temple Street, Suite 3D

New Haven, CT 06510 (USA)

E-Mail emilyli019@gmail.com 
around medium-sized vessels with an epithelioid endothelial cell lining and are surrounded by associated lymphocytic and eosinophilic infiltrates $[1,2]$. The tumors typically present in the head and neck, especially in the preauricular area and on the scalp [2-6]. Systemic involvement is infrequent but can include lymphadenopathy, peripheral eosinophilia, and arteriovenous shunts [4]. The majority of affected patients are female (70\%), in their third to fifth decade of life, and can be of any racial background $[3,4]$.

ALHE can mimic a range of conditions and impose a diagnostic challenge $[1,2,7]$. Vascular tumors that can present similarly include Kimura disease, epithelioid hemangioendothelioma, angiosarcoma, eosinophilic granulomatosis with polyangiitis, and juvenile temporal arteritis (JTA). Additional diagnoses to consider when evaluating subcutaneous head lesions are Kaposi's sarcoma, metastasis, lymphoma, and pyogenic granuloma [4]. Similar and overlapping clinical features among these disorders necessitate histopathological evaluation to make a definitive diagnosis. Herein, we present a case of a pediatric superotemporal brow mass suspected to be JTA prior to excisional biopsy. Review of histopathology refuted the presumed diagnosis and highlights the necessity for awareness of atypical presentations of ALHE.

\section{Case Report}

A 20-year-old boy with a history of Hashimoto's thyroiditis and papillary thyroid carcinoma who previously underwent total thyroidectomy and adjunctive radioactive iodine thyroid ablation initially presented with a mass located superotemporal to his right eyebrow. The mass had developed 1 month prior and initially enlarged and became erythematous before stabilizing in size with resolution of the erythema. He denied pruritus, tenderness, drainage, and additional lesions. He sustained no preceding trauma to the area. Examination revealed a $1.5-\mathrm{cm}$ mobile, nontender subcutaneous lesion above the superotemporal aspect of the right eyebrow without palpable or visible overlying pulsation (Fig. 1a). Workup at the time included complete blood count, thyroid function tests, and thyroglobulin panel, which were all unremarkable. The lesion was thought to be a benign tumor or cyst.

At follow-up 6 months after the initial evaluation, the patient remained asymptomatic, and the mass was unchanged in appearance. However, examination demonstrated a palpable pulse over the lesion. Doppler ultrasound ruled out lymphadenopathy and a dural cyst but revealed a hypoechoic ring around the vessel lumen, representing localized thickening of the right superficial temporal artery wall, which was suggestive of arteritis (Fig. 2) [8]. Further review of systems was negative for fatigue, jaw claudication, scalp tenderness, decreased appetite, and fluctuating vision. Given the patient's strong family history of inflammatory disor-
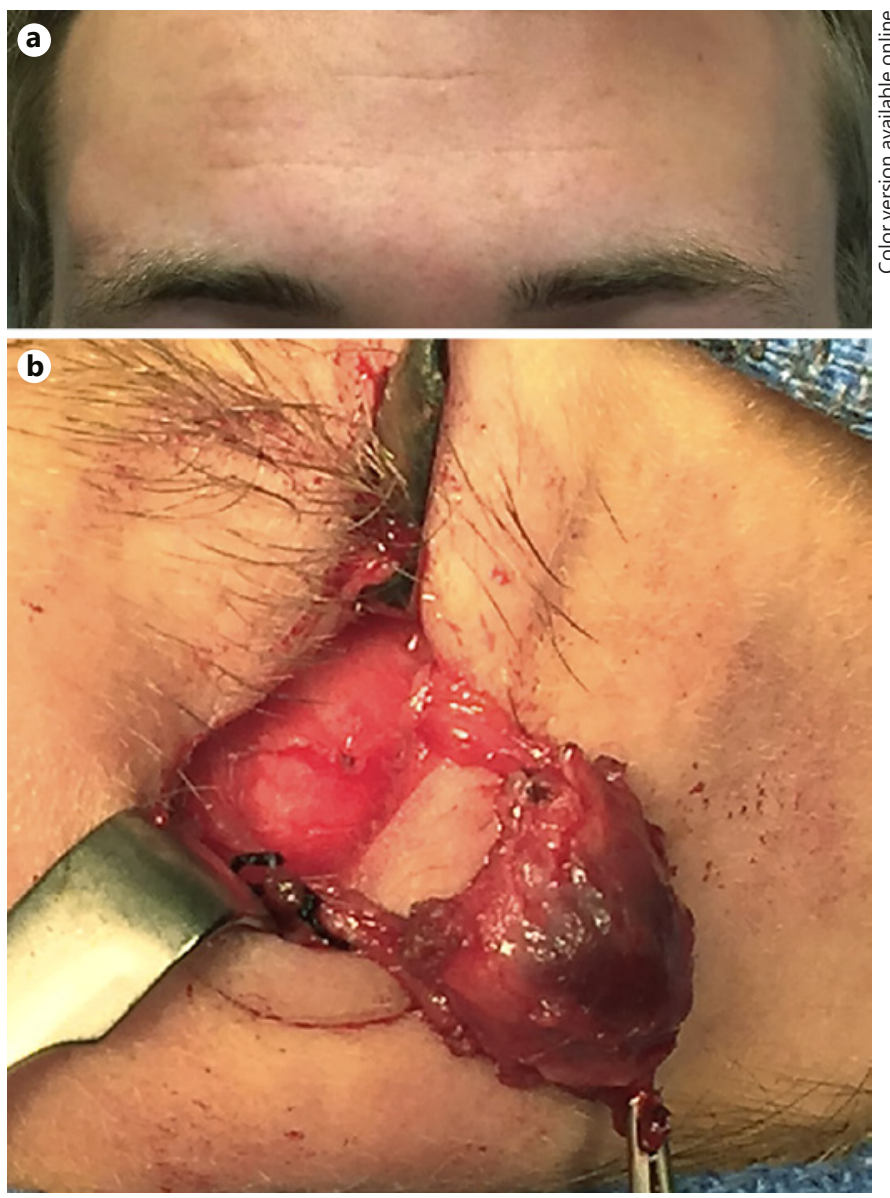

Fig. 1. External color photographs of the mass. a Clinical appearance of the right temporal mass prior to surgical excision. b In situ appearance of the lesion during surgical exploration. There were two nonpulsatile stalks extending from the mass.

ders (systemic lupus erythematosus, Hashimoto's thyroiditis, and uveitis), the patient underwent serological evaluation by rheumatology. Testing was weakly positive for antinuclear antibody (1:80 homogenous) and negative for rheumatoid factor, anticyclic citrullinated peptide antibodies, and antineutrophil cytoplasmic antibodies. Complete metabolic panel, complete blood count, erythrocyte sedimentation rate, and C-reactive protein were normal.

The leading diagnosis was JTA, and he was referred to oculoplastic surgery for a temporal artery biopsy. Prior to excision, magnetic resonance imaging of the brain with contrast demonstrated a prominent right superficial temporal artery with adjacent soft tissue thickening and enhancement with gadolinium, suggesting a diagnosis of temporal arteritis (Fig. 3) [8]. The patient underwent surgical excision; operative exploration revealed a $1.2 \times 0.9 \times 0.1$ $\mathrm{cm}$ nodule with two nonpulsatile stalks (Fig. $1 \mathrm{~b}$ ).

After fixation and embedding in paraffin, the hematoxylin and eosin-stained sections revealed an atherosclerotic temporal artery with marked intimal thickening from loose fibrous tissue surrounded by a proliferation of lymphoid tissue, blood vessels, and 

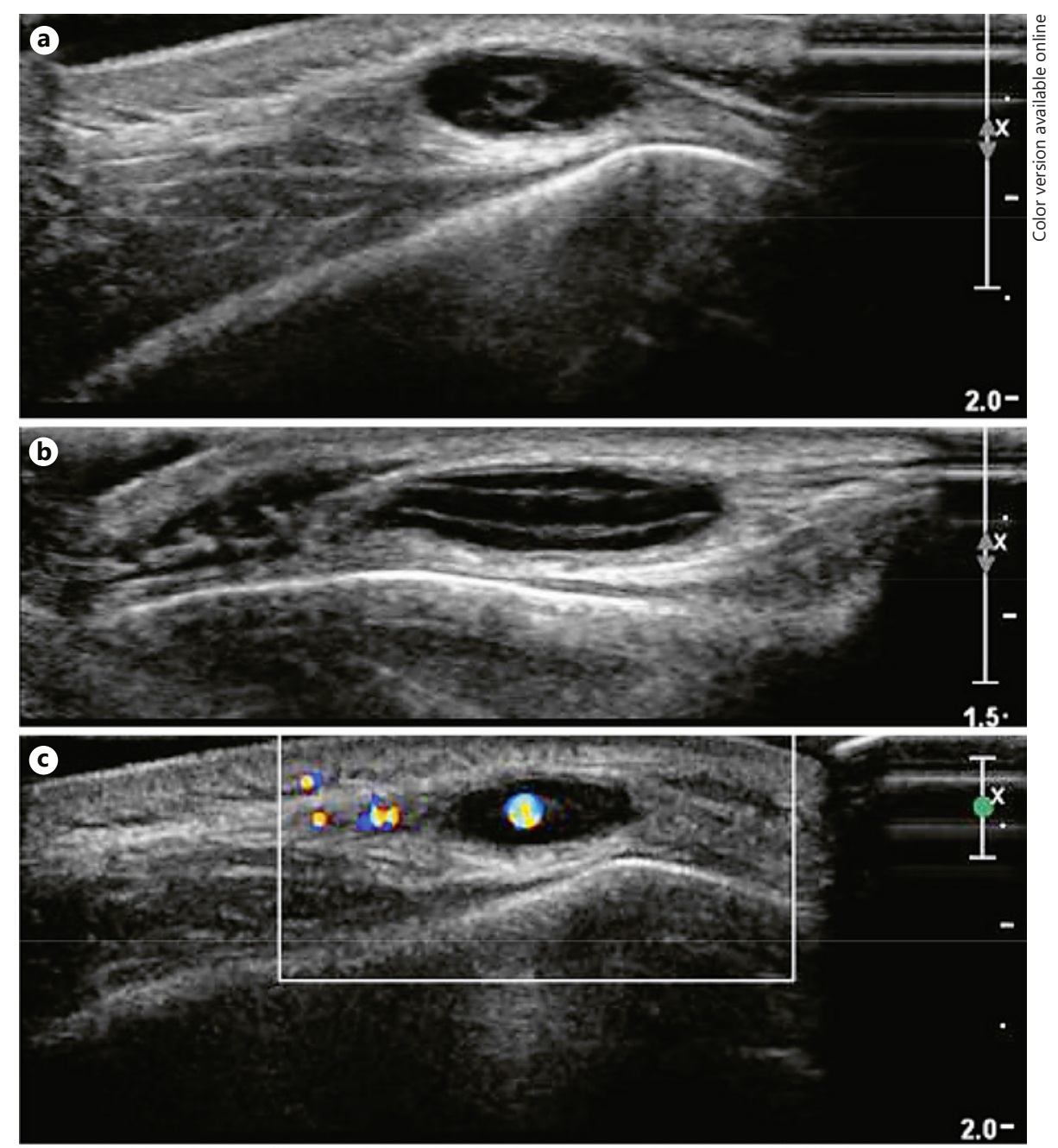

Fig. 2. Ultrasound of the right superficial temporal artery demonstrating focal edema and vessel wall thickening. a Cross-sectional view. b Lateral view. Doppler demonstrates vessel patency and normal flow velocity. c Cross-sectional view. d Lateral view.

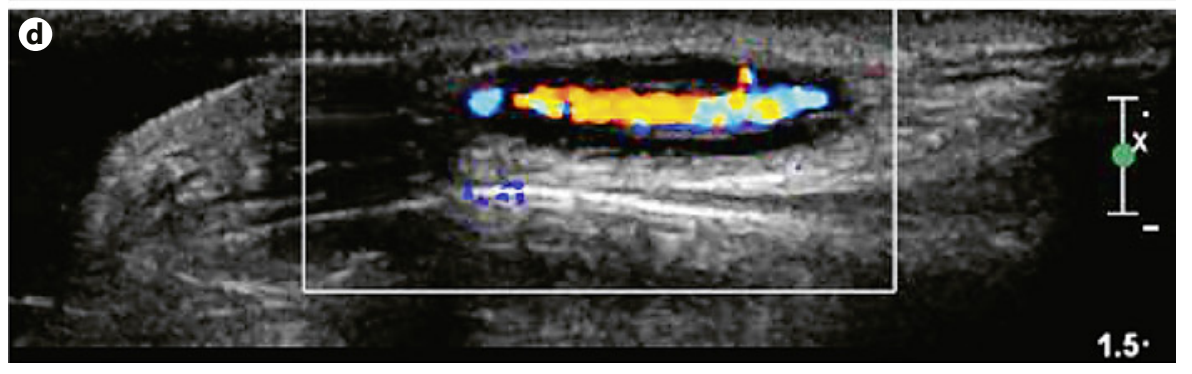

endothelial cells. Over an arc comprising 25\% of the vessel circumference, the internal elastic lamina, highlighted by an elastic stain, and the muscular wall were absent, suggesting prior vasculitis or injury. There was no active inflammation, necrosis, or fibrosis. The remaining muscular wall showed an orientation change from circumferential to longitudinal muscle fibers. Angiolymphoproliferative tissue, plump epithelioid cells, eosinophils, mast cells, and foci of necrosis surrounded the vessel (Fig. 4). CD31 staining highlighted endothelial proliferation. No significant mitotic rate was appreciated. Fungal and acid-fast organism stains were negative. The final histopathological diagnosis was ALHE.

Angiolymphoid Hyperplasia with Eosinophilia

\section{Discussion/Conclusion}

Inflammatory vascular tumors of the head are uncommon, poorly characterized in the pediatric population, and can be difficult to diagnose. A number of mimickers have obscured the diagnosis of ALHE $[1,2,7]$. To the best of our knowledge, only one other case of ALHE resembling temporal arteritis exists in the literature. Kitamura et al. [1] reported a 68-year-old Japanese woman who presented with a painful, pulsatile temporal mass that was

Ocul Oncol Pathol 2020;6:25-30 

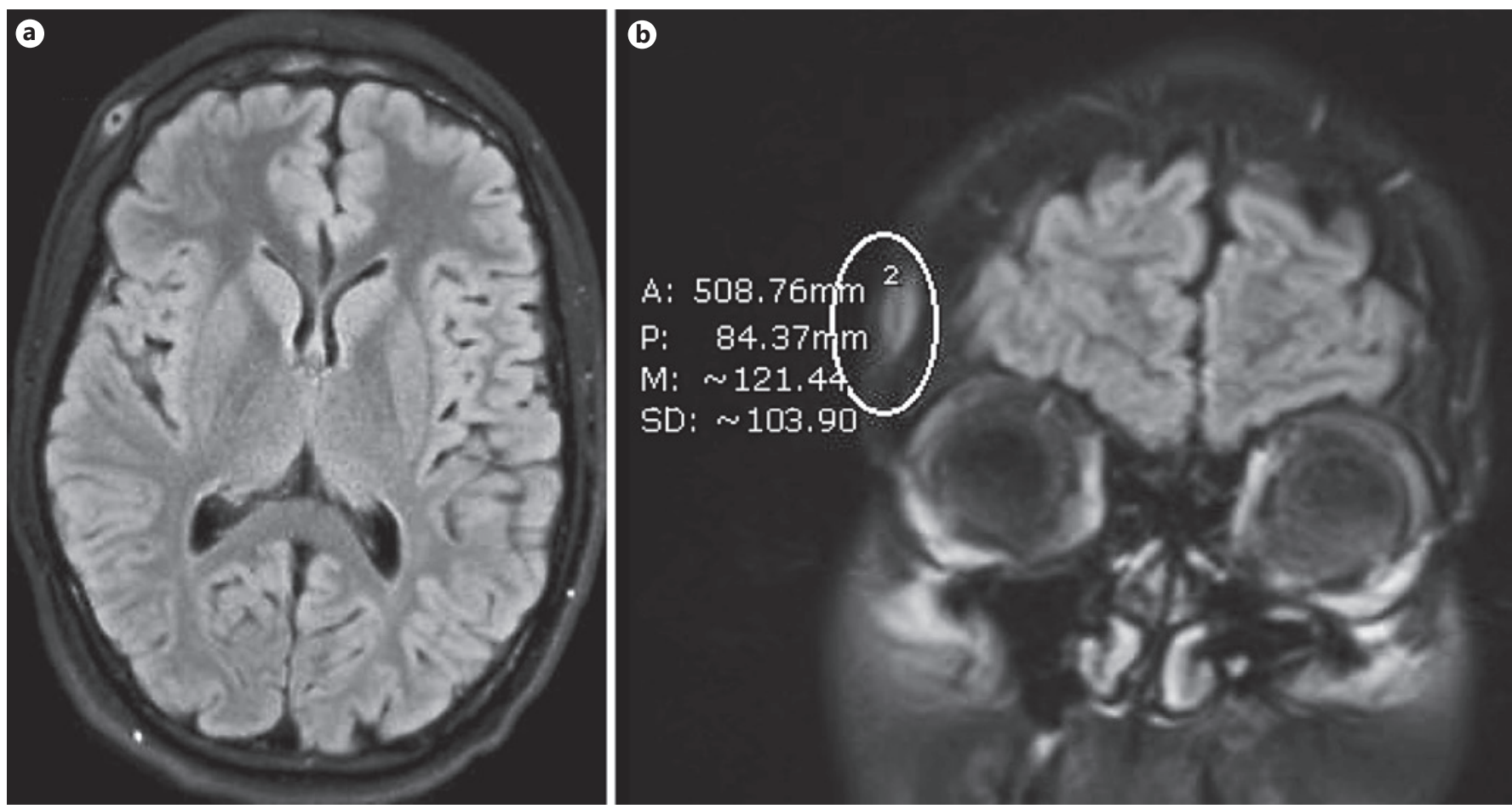

Fig. 3. Magnetic resonance imaging showing prominence of the right superficial temporal artery and adjacent soft tissue thickening consistent with temporal arteritis. a Axial section. b Coronal section.

undetected on a computed tomography scan of the head before excisional biopsy demonstrated the diagnosis of ALHE. Our patient's atypical demographic, clinical, and radiographic features broaden the scope of ALHE characterization in literature.

Clinical and radiographic appearance in our case of ALHE suggested JTA before histopathological evaluation made the definitive diagnosis. Whereas ALHE typically affects young to middle-aged women, JTA occurs in older children and young adults (age $<50$ years) $[3,4,9]$. Both characteristically manifest as a painless mass, though the most common locations differ between the two - the preauricular area or the scalp in ALHE versus the temporal artery distribution in JTA $[3,6,10]$. Erythrocyte sedimentation rate is normal in JTA and ALHE, and there may be peripheral eosinophilia in ALHE but not in JTA $[9,10]$. Histopathologically, ALHE has prominent neoangiogenesis, epithelioid endothelial cells, eosinophils, lymphoid follicles, and fibrosis [3, 4]. In contrast, while JTA presents as a localized temporal mass, its histopathological appearance includes arterial wall intimal thickening in addition to lymphohistiocytic infiltrate with or without giant cells and eosinophilia $[1,10]$.
Evidence of endothelial proliferation, plump epithelioid cells, eosinophils, and mast cells in our case led to the diagnosis of ALHE with clinical features that overlapped with JTA.

An evolving, undefined spectrum of vascular tumors that encompasses ALHE makes the diagnosis of ALHE all the more challenging. ALHE may be more commonly referred to as EH, or it may represent a distinct subtype of $\mathrm{EH}$ [11]. Kimura disease, a vasoproliferative condition of the head and neck that may lie within this range, contains significant overlap with ALHE but is distinguished by demographic and histological features [11, 12]. Although Kimura disease typically presents with head and neck skin lesions in young males with autoimmune predisposition, its histology is defined by a predominance of lymphoid follicles and eosinophilic inflammation. In contrast, ALHE tends to occur in young to middle-aged females, and significant vascular proliferation of epithelioid endothelial cells differentiates ALHE from Kimura disease on histology $[1,12]$.

Despite extensive preceding evaluation, the diagnosis in our case of ALHE presenting with clinical features of JTA was elucidated by histopathology, underscoring the 


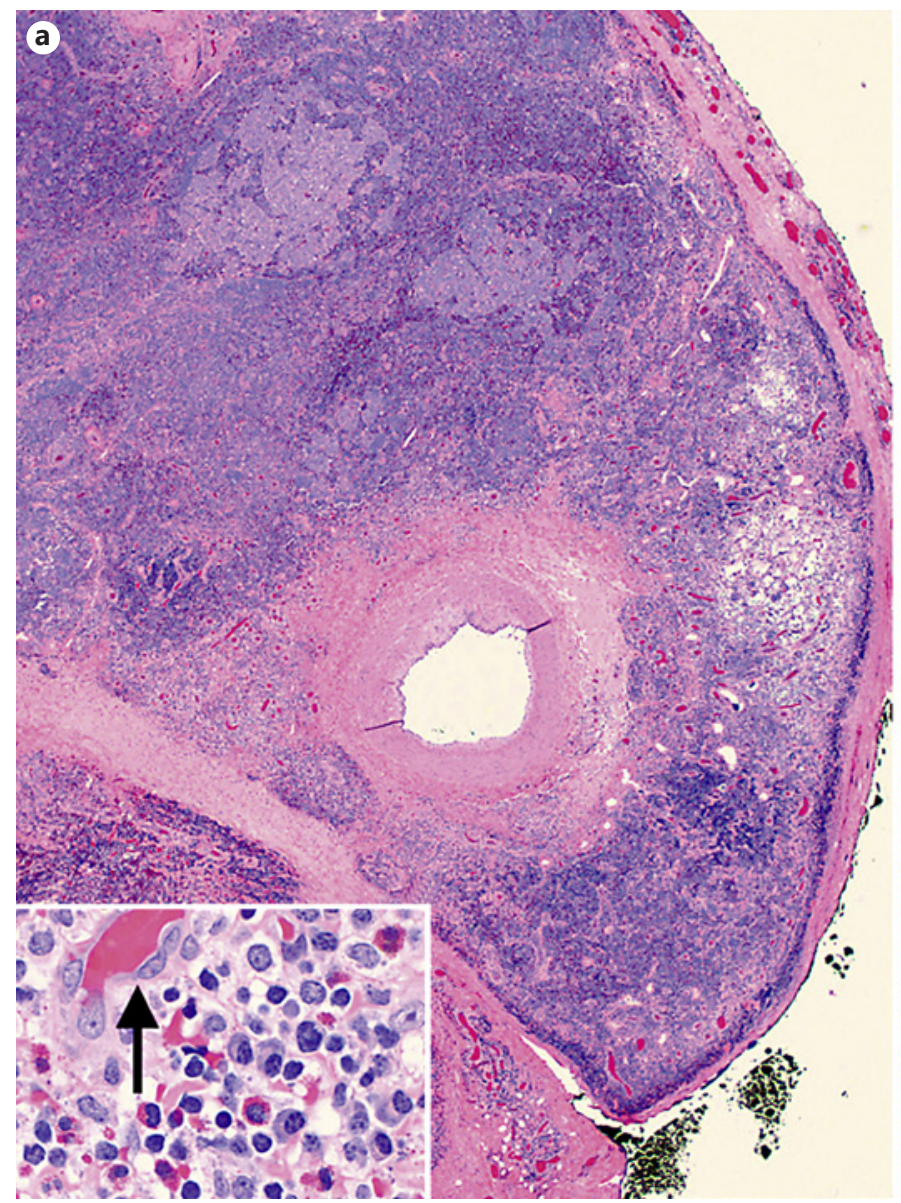

Fig. 4. Histopathological analysis. a Hematoxylin and eosinstained slide showing the temporal artery and surrounding angiolymphoid infiltrate with germinal centers $(\times 2)$. Inset Higher magnification reveals numerous eosinophils and mast cells within the

significant clinical overlap and need for continued characterization of temporal vascular tumors in the pediatric population.

\section{Statement of Ethics}

The authors have no ethical conflicts to disclose.

\section{Disclosure Statement}

The authors have no conflicts of interest to declare.

\section{Funding Sources}

The present study did not have any sources of funding.

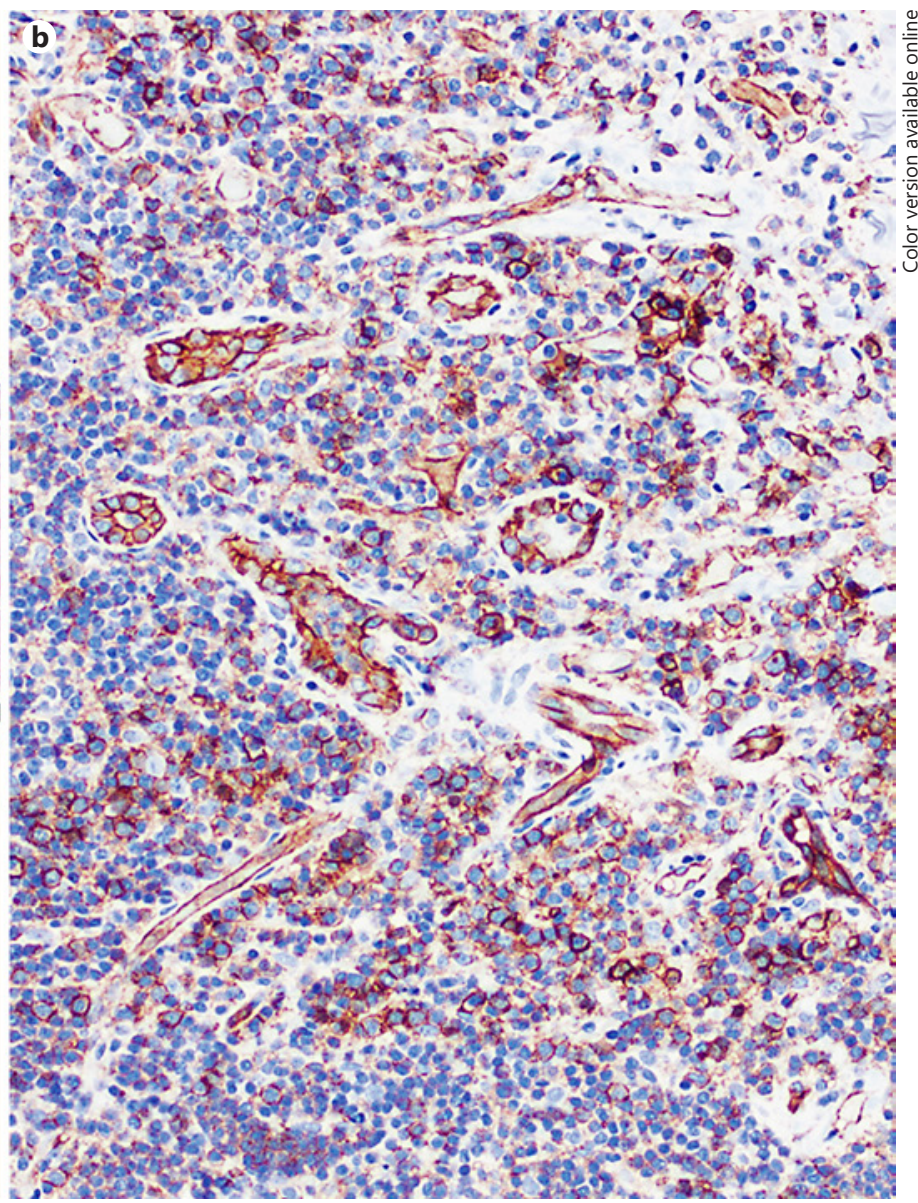

lesion. The black arrow points to epithelioid endothelial cells lining a vessel $(\times 60)$. b CD31 immunostain highlights the proliferation of vessels lined by plump endothelial cells $(\times 20)$.

\section{Author Contributions}

E.L., J.S., A.D., and M.S. participated in the conception and design, acquisition of data, and analysis and interpretation of data. E.L. was responsible for drafting the manuscript. E.L., J.S., A.D., and M.S. participated in revising the manuscript for intellectual content. E.L., J.S., A.D., and M.S. gave final approval of the completed manuscript.

\section{References}

Ocul Oncol Pathol 2020;6:25-30 DOI: $10.1159 / 000500361$
1 Kitamura H, Ito S, Kuwana N, Yutani C. Epithelioid hemangioma of the temporal artery clinically mimicking temporal arteritis. Pathol Int. 1999 Sep;49(9):831-5.

2 Ahmad SM, Wani GM, Khursheed B, Qayoom S. Angiolymphoid hyperplasia with eosinophilia mimicking multiple cylindromas: a rare case report. Indian J Dermatol. 2014 Jul; 59(4):423. 
3 Moira R, Giuseppe F, Riccardo V, Nicola R, Daniele B, Pierfrancesco C, et al. Epithelioid hemangioma of brachial artery: report of a case and review of the literature. Open Med (Wars). 2015 Dec;10(1):502-7.

4 Fite-Trepat L, Martos-Fernandez M, Alberola-Ferranti M, Pablo-Garcia-Cuenca A, Bescosatin C. Angiolymphoid Hyperplasia with Eosinophilia Involving the Occipital Artery: Case Report and Review of Literature. J Clin Diagn Res. 2017 Mar;11(3):ZD21-3.

5 Azari AA, Kanavi MR, Lucarelli M, Lee V, Lundin AM, Potter HD, et al. Angiolymphoid hyperplasia with eosinophilia of the orbit and ocular adnexa: report of 5 cases. JAMA Ophthalmol. 2014 May;132(5):633-6.
6 Tauziède-Espariat A, Raffoul J, Sun SR, Lassabe C, Monnin C. [A cutaneous case of angiolymphoid hyperplasia with eosinophilia: presentation of a rare entity]. Ann Pathol. 2018 Dec;38(6):386-90.

7 Hamaguchi Y, Fujimoto M, Matsushita Y, Kitamura-Sawada S, Kawano M, Takehara K. IgG4-related skin disease, a mimic of angiolymphoid hyperplasia with eosinophilia. Dermatology. 2011;223(4):301-5.

8 Younger DS. Giant Cell Arteritis. Neurol Clin. 2019 May;37(2):335-44

9 Czihal M, Tato F, Hoffmann U, Kuhlencordt PJ. Juvenile temporal arteritis. Volume 31. Italy: Clin Exp Rheumatol; 2013. p. S89.
10 Andonopoulos AP, Melachrinou M, Yiannopoulos G, Meimaris N. Juvenile temporal arteritis: a case report and review of the literature. Clin Exp Rheumatol. 2004 May-Jun; 22(3):379-80.

11 Huang SC, Zhang L, Sung YS, Chen CL, Krausz T, Dickson BC, et al. Frequent FOS Gene Rearrangements in Epithelioid Hemangioma: A Molecular Study of 58 Cases With Morphologic Reappraisal. Am J Surg Pathol. 2015 Oct;39(10):1313-21.

12 Marka A, Cowdrey MC, Carter JB, Lansigan F, Yan S, LeBlanc RE. Angiolymphoid hyperplasia with eosinophilia and Kimura disease overlap, with evidence of diffuse visceral involvement. J Cutan Pathol. 2019 Feb;46(2):138-42. 